\title{
Anti-Helicobacter pylori Activity of the Methanolic Extract of Geum iranicum and its Main Compounds
}

\author{
Somayeh Shahani ${ }^{\mathrm{a}}$, Hamid R. Monsef-Esfahani ${ }^{\mathrm{a}}$, Soodabeh Saeidnia ${ }^{\mathrm{b}}$, \\ Parastoo Saniee ${ }^{\mathrm{c}}$, Farideh Siavoshic, Alireza Foroumadi ${ }^{\mathrm{d}}$, Nasrin Samadie, \\ and Ahmad R. Gohari ${ }^{\mathrm{b}, *}$ \\ a Department of Pharmacognosy, Faculty of Pharmacy, Tehran University of Medical \\ Sciences, Tehran, Iran \\ b Medicinal Plants Research Center, Tehran University of Medical Sciences, Tehran, \\ P. O. Box 14155-6451, Iran. Fax: +98-21-64122330. E-mail: goharii@tums.ac.ir \\ c Microbiology Department, Faculty of Sciences, University of Tehran, Tehran, Iran \\ d Pharmaceutical Sciences Research Center, Tehran University of Medical Sciences, \\ Tehran, Iran \\ e Department of Drug and Food Control, Faculty of Pharmacy and Biotechnology \\ Research Center, Tehran University of Medical Sciences, Tehran, Iran \\ * Author for correspondence and reprint requests \\ Z. Naturforsch. 67 c, 172-180 (2012); received May 30, 2011/January 14, 2012
}

Geum iranicum Khatamsaz, belonging to the Rosaceae family, is an endemic plant of Iran. The methanol extract of the roots of this plant showed significant activity against one of the clinical isolates of Helicobacter pylori which was resistant to metronidazole. The aim of this study was the isolation and evaluation of the major compounds of G. iranicum effective against $H$. pylori. The compounds were isolated using various chromatographic methods and identified by spectroscopic data $\left({ }^{1} \mathrm{H}\right.$ and ${ }^{13} \mathrm{C}$ NMR, HMQC, HMBC, EI-MS). An antimicrobial susceptibility test was performed employing the disk diffusion method against clinical isolates of $H$. pylori and a micro dilution method against several Gram-positive and Gram-negative bacteria; additionally the inhibition zone diameters (IZD) and minimum inhibitory concentrations (MIC) values were recorded. Nine compounds were isolated: two triterpenoids, uvaol and niga-ichigoside $\mathrm{F} 1$, three sterols, $\beta$-sitosterol, $\beta$-sitosteryl acetate, and $\beta$-sitosteryl linoleate, one phenyl propanoid, eugenol, one phenolic glycoside, gein, one flavanol, (+)-catechin, and sucrose. The aqueous fraction, obtained by partitioning the $\mathrm{MeOH}$ extract with water and chloroform, was the most effective fraction of the extract against all clinical isolates of $H$. pylori. Further investigation of the isolated compounds showed that eugenol was effective against $H$. pylori but gein, diglycosidic eugenol, did not exhibit any activity against $H$. pylori. The subfraction $\mathrm{D}_{4}$ was the effective fraction which contained tannins. It appeared that tannins were probably the active compounds responsible for the anti-H. pylori activity of $G$. iranicum. The aqueous fraction showed a moderate inhibitory activity against both Gram-positive and Gram-negative bacteria. The MIC values indicated that Gram-positive bacteria including Staphylococcus aureus, Staphylococcus epidermidis, and Bacillus subtilis are more susceptible than Gram-neagative bacteria including Escherichia coli and Pseudomonas aeruginosa.

Key words: Geum iranicum, Helicobacter pylori, Eugenol

\section{Introduction}

Helicobacter pylori colonizes the stomachs of about $50 \%$ of the world's human population. This organism is the main risk factor for peptic ulceration as well as gastric mucosal-associated lymphoid tissue (MALT) lymphoma and gastric adenocarcinoma. The prevention of $H$. pylori colonization could potentially provide primary prevention of the mentioned diseases (Fauci et al., 2008). The common treatments of $H$. pylori infection, including antibiotics and a proton pump inhibitor, may fail for several reasons. The main reason was found to be $H$. pylori resistance to antibiotics like clarithromycin and metronidazole (Mégraud, 2004). Therefore, the research for new anti-H. pylori drugs from plant sources is ongoing (Nariman et al., 2004).

The genus Geum, belonging to the Rosaceae family, is a perennial rhizomatous herb with five species in Iran of which G. iranicum Khatamsaz is an endemic one (Khatamsaz, 1992; Mozaffarian, 
1996). Some Geum species are used as medicinal plants in folk medicine (Vollmann and Schultze, 1995; Gruenwald, 2004). The roots of G. urbanum and $G$. rivale are employed against diarrhea (Gruenwald, 2004), and G. japonicum has been used as diuretic and astringent in traditional Chinese medicine and Japanese folk medicine (Ming et al., 2000). In an Iranian folk remedy, the infusion of the root of $G$. iranicum is employed to treat gastrointestinal disorders like diarrhea, and a decoction of the whole plant is combined with wheat flour and used as a poultice for frostbite (Abutorabi, 2001). Previous studies on extracts of Geum species have shown that they can be effective in the treatment of some diseases. A new triterpene acid, $2 \alpha, 19 \alpha$-dihydroxy-3-oxo-urs-12en-28-oic acid, from the extract of $G$. japonicum showed potent inhibitory activity against HIV-1 protease (Xu et al., 2000). In addition, the extract of G. japonicum was active against HSV-1 and HSV-2 (herpes simplex virus) (Kurokawa et al., 1995) and CMV (cytomegalo virus) (Yukawa et al., 1996). Some tannins isolated from the extract of this plant showed potent anticoagulant activity (Zeng et al., 1998). Other Geum species showed antioxidant (Russo et al., 2005), antimicrobial (Panizzi et al., 2000), and anti-inflammatory (Tunón et al., 1995) effects, respectively. In another study, antimicrobial activity was reported for the extract and essential oil of Geum kokanicum that was collected in Iran (Faramarzi et al., 2008). Furthermore, the polar extract of this species showed a potent inhibitory effect on matrix metalloproteinase activity at minimal cytotoxic doses (Khorramizdeh et al., 2006).

A literature review revealed that the antiH. pylori activity of Geum species has not been investigated. In the present study we aimed therefore to evaluate the activity of various extracts, sub-fractions, and main components of $G$. iranicum against clinical isolates of $H$. pylori (resistant to metronidazole). Furthermore, the evaluation of the antibacterial activity (against Gram-positive and Gram-negative bacteria) of fractions, together with the isolation and identification of the separated compounds, is reported.

\section{Material and Methods}

\section{General experimental procedures}

Silica gel $60 \mathrm{~F}_{254}$ pre-coated plates (Merck, Darmstadt, Germany) and anisaldehyde $/ \mathrm{H}_{2} \mathrm{SO}_{4}$ reagent followed by heating were used for detecting the compounds. NMR experiments were performed on a Bruker Avance 500 DRX $(500 \mathrm{MHz}$ for ${ }^{1} \mathrm{H}$ and $125 \mathrm{MHz}$ for ${ }^{13} \mathrm{C}$ ) spectrometer (Rheinstetten, Germany). EI-MS spectra were measured on an Agilent Technology (Palo Alto CA, USA) instrument with a 5973 Network mass selective detector (MS model). A $\mathrm{CO}_{2}$ incubator (Heraeus, Hamburg, Germany) was used for the antibacterial susceptibility tests.

\section{Plant collection}

Roots of Geum iranicum were collected from the Gloul Sarani protected area, $75 \mathrm{~km}$ north of Shirvan, province of Khorasan-e-Shomali, Iran, at $2460 \mathrm{~m}$ above sea level, during the flowering stage in June 2009. A voucher specimen (6714 THE) was deposited at the herbarium of the Faculty of Pharmacy, Tehran University of Medical Sciences, Tehran, Iran.

\section{Antimicrobial susceptibility test}

Three clinical isolates of $H$. pylori, Is.1, Is.2, and Is.3, were obtained from patients with chronic gastritis who had been referred to the Endoscopy Unit at Shariati Hospital, Tehran, Iran. Antral biopsies with positive rapid urease tests were cultured in the microbiology laboratory as described in our previous study (Siavoshi et al., 2010). Antimicrobial susceptibility was tested by the disk diffusion method according to the guidelines of the NCCLS (2006). Serial dilutions of test samples were made in dimethyl sulfoxide (DMSO) $(100,50,25,12.5 \mu \mathrm{g} / \mathrm{ml})$. Bacterial suspensions were prepared in normal saline with the turbidity of McFarland standard No. 2 (equivalent to $6 \cdot 10^{8}$ cell $/ \mathrm{ml}$ ). The surface of blood agar plates was inoculated with $100 \mu$ l of each bacterial suspension. Then, plates were dried at room temperature for about $10 \mathrm{~min}$. The sterile blank disks $(6 \mathrm{~mm}$ in diameter) were placed on the surface of inoculated plates and impregnated with $10 \mu \mathrm{l}$ of each sample dilution. Control plates included blank disks impregnated with $10 \mu \mathrm{l}$ of DMSO. Plates were incubated at $37^{\circ} \mathrm{C}$ under microaerobic conditions and examined after $3-5 \mathrm{~d}$. The inhibition zone diameters (IZD) were recorded.

Antibacterial activity of the aqueous fraction was also tested against several Gram-positive and Gram-negative bacteria including Staphylococcus aureus ATCC 6538, Staphylococcus epider- 
midis ATCC 12228, Bacillus subtilis ATCC 6633, Escherichia coli ATCC 8739, and Pseudomonas aeruginosa ATCC 9027 by a micro dilution method using 96 U-shaped wells plates (NCCLS, 2006). A $200-\mu \mathrm{l}$ aliquot of stock solutions of the fraction $(128 \mathrm{mg} / \mathrm{ml})$ and ciprofloxacin $(100 \mu \mathrm{g} / \mathrm{ml})$ as a standard antibiotic compound in Mueller-Hinton broth (MHB) were transferred into the first well in each row and serially diluted by mixing with $100 \mu \mathrm{l}$ of MHB in subsequent wells. Then $100 \mu \mathrm{l}$ of bacterial suspension $\left(1 \cdot 10^{6} \mathrm{CFU} / \mathrm{ml}\right)$ were added to each well to reach the final inoculum size of about $5 \cdot 10^{5} \mathrm{CFU} / \mathrm{ml}$. After $24 \mathrm{~h}$ of incubation at $35^{\circ} \mathrm{C}$, the microdilution trays were tested for the absence or presence of visible growth. The endpoint minimum inhibitory concentration (MIC) is the lowest concentration of the fraction at which the test strain does not demonstrate visible growth.

\section{Extraction and isolation procedure}

Dried roots of G. iranicum $(1 \mathrm{~kg})$ were cut into small pieces and extracted with EtOAc and $\mathrm{MeOH}$ ( $3 \times 41$ for each solvent), successively, by percolation at room temperature to obtain EtOAc $(6 \mathrm{~g})$ and $\mathrm{MeOH}(75 \mathrm{~g})$ extracts. The EtOAc extract was subjected to silica gel column chromatography (CC) $(4 \times 20 \mathrm{~cm})$ using $n$-hexane $/ \mathrm{CHCl}_{3}(7: 3$, 3:7 $\mathrm{v} / \mathrm{v})$, EtOAc, and $\mathrm{MeOH}$ as eluents to give 7 fractions $(A-G)$. Fraction A was further separated by silica gel CC $(3 \times 20 \mathrm{~cm})$ with $n$-hexane, $n$-hexane/ $\mathrm{CHCl}_{3}(7: 3,3: 7 \mathrm{v} / \mathrm{v})$ and $\mathrm{CHCl}_{3}$ to give 5 fractions $\left(\mathrm{A}_{1}-\mathrm{A}_{5}\right)$ of which fraction $\mathrm{A}_{3}$ contained compound $1(15 \mathrm{mg})$. Silica gel CC $(2 \times 20 \mathrm{~cm})$ was used for separation of fraction $\mathrm{B}$ to yield 7 fractions $\left(\mathrm{B}_{1}-\mathrm{B}_{7}\right)$ with $n$-hexane/ $\mathrm{CHCl}_{3}(7: 3,6: 4$, 4:6 v/v) and $\mathrm{CHCl}_{3}$. Fraction $\mathrm{B}_{2}$ was $2(22 \mathrm{mg})$. Fraction $\mathrm{C}$ was fractionated with $n$-hexane $/ \mathrm{CHCl}_{3}$ $(9: 1,5: 5 \mathrm{v} / \mathrm{v})$ by silica gel CC $(2 \times 20 \mathrm{~cm})$ to give fractions $\mathrm{C}_{1}-\mathrm{C}_{5}$; compound 3 (141 mg) was isolated from fraction $\mathrm{C}_{4}$. Compound $4(5 \mathrm{mg})$ was obtained from fraction $\mathrm{D}$ using silica gel CC $(2.5 \times 20 \mathrm{~cm})$ with $n$-hexane/EtOAc $(8: 2 \mathrm{v} / \mathrm{v})$.

The $\mathrm{MeOH}$ extract $(75 \mathrm{~g})$ was suspended in water and extracted with $\mathrm{CHCl}_{3}$ to obtain $\mathrm{CHCl}_{3}$ and aqueous fractions. The $\mathrm{CHCl}_{3}$ fraction was further chromatographed with $n$-hexane $/ \mathrm{CHCl}_{3}(7: 3,3: 7$ $\mathrm{v} / \mathrm{v})$ and $\mathrm{CHCl}_{3}$ to give 6 fractions of which fraction 5 was compound $5(17 \mathrm{mg})$. The aqueous portion of the $\mathrm{MeOH}$ extract $(70 \mathrm{~g})$ was subjected to silica gel CC $(10 \times 20 \mathrm{~cm})$ with EtOAc, EtOAc/
$\mathrm{MeOH}(9: 1,7: 3,4: 6 \mathrm{v} / \mathrm{v})$ and $\mathrm{MeOH}$ to yield 6 fractions $(\mathrm{A}-\mathrm{F})$. Fraction A was separated by silica gel $\mathrm{CC}(2.5 \times 20 \mathrm{~cm})$ with $\mathrm{CHCl}_{3} / \mathrm{MeOH}$ $(19: 1,9: 1,8: 2 \mathrm{v} / \mathrm{v})$ to yield 5 fractions $\left(\mathrm{A}_{1}-\mathrm{A}_{5}\right)$. Fraction $\mathrm{A}_{4}$ was compound 6 (129 mg). Fraction C was subjected to silica gel CC $(2.5 \times 20 \mathrm{~cm})$ with $\mathrm{CHCl}_{3} / \mathrm{MeOH}(8: 2,7: 3 \mathrm{v} / \mathrm{v})$ to give 4 fractions $\left(\mathrm{C}_{1}-\mathrm{C}_{4}\right)$. Compound 7 (13 mg) was obtained from $\mathrm{C}_{2}$ using silica gel $\mathrm{CC}(1.5 \times 40 \mathrm{~cm})$ with $\mathrm{CHCl}_{3} /$ $\mathrm{MeOH}(75: 25 \mathrm{v} / \mathrm{v})$. Fraction D was chromatographed on a silica gel column $(4 \times 20 \mathrm{~cm})$ with $\mathrm{CHCl}_{3} / \mathrm{MeOH}(8: 2,6: 4 \mathrm{v} / \mathrm{v})$ and $\mathrm{MeOH}$ to result in factions $\mathrm{D}_{1}-\mathrm{D}_{4}$ of which $\mathrm{D}_{2}$ was compound 8 (70 mg). Compound 9 (960 mg) was obtained from fraction E using silica gel CC $(2.5 \times 20 \mathrm{~cm})$ with EtOAc/MeOH (6:4 v/v).

$\beta$-Sitosteryl acetate (1): $R_{\mathrm{f}}=0.14$ in $n$-hexane/ $\mathrm{CHCl}_{3}(7: 3 \mathrm{v} / \mathrm{v}) .-{ }^{1} \mathrm{H}$ NMR $\left(\mathrm{CDCl}_{3}, 500 \mathrm{MHz}\right)$ : $\delta_{\mathrm{H}}=0.69(3 \mathrm{H}, \mathrm{s}, \mathrm{H}-18), 0.82(3 \mathrm{H}, \mathrm{d}, J=6.8 \mathrm{~Hz}$, $\mathrm{H}-27), 0.84(3 \mathrm{H}, \mathrm{d}, J=6.8 \mathrm{~Hz}, \mathrm{H}-26), 0.86(3 \mathrm{H}, \mathrm{t}$, $J=7.3 \mathrm{~Hz}, \mathrm{H}-29), 0.93(3 \mathrm{H}, \mathrm{d}, J=6.5 \mathrm{~Hz}, \mathrm{H}-21)$, $1.03(3 \mathrm{H}, \mathrm{s}, \mathrm{H}-19), 2.05,\left(3 \mathrm{H}, \mathrm{s}, \mathrm{OCH}_{3}\right), 4.61(1 \mathrm{H}$, $\mathrm{m}, \mathrm{H}-3), 5.38(1 \mathrm{H}$, brd, $J=5 \mathrm{~Hz}, \mathrm{H}-6) \cdot-{ }^{13} \mathrm{C} \mathrm{NMR}$ $\left(\mathrm{CDCl}_{3}, 500 \mathrm{MHz}\right): \delta_{\mathrm{C}}=11.8(\mathrm{C}-18), 11.9(\mathrm{C}-29)$, 18.8 (C-21), 19.0 (C-27), 19.3 (C-19), 19.8 (C-26), 21.0 (C-11), $21.4\left(\mathrm{COOCH}_{3}\right), 23.1(\mathrm{C}-28), 24.3$ (C-15), 26.1 (C-23), 27.7 (C-2), 28.2 (C-16), 29.1 (C-25), 31.8 (C-8), 31.9 (C-7), 33.9 (C-22), 36.1 (C-20), 36.6 (C-10), 36.9 (C-1), 38.1 (C-4), 39.7 (C-12), 42.3 (C-13), 45.8 (C-24), 50.0 (C-9), 56.0 (C-17), 56.7 (C-14), 73.9 (C-3), 122.6 (C-6), 139.6 (C-5), $170.5(\mathrm{C}=\mathrm{O})$. - EI-MS: $\mathrm{m} / z(\%)=396[\mathrm{M}-$ $\mathrm{HOAc}^{+}$(100), 381 (15), 288 (9), 275 (6), 255 (18), 213 (21).

$\beta$-Sitosteryl linoleate (2): $R_{\mathrm{f}}=0.37$ in $n$-hexane/ $\mathrm{CHCl}_{3}(7: 3 \mathrm{v} / \mathrm{v}) .-{ }^{1} \mathrm{H}$ NMR $\left(\mathrm{CDCl}_{3}, 500 \mathrm{MHz}\right)$ : $\delta_{\mathrm{H}}=0.68(3 \mathrm{H}, \mathrm{s}, \mathrm{H}-18), 0.81(3 \mathrm{H}, \mathrm{d}, J=7 \mathrm{~Hz}, \mathrm{H}-27)$, $0.83(3 \mathrm{H}, \mathrm{d}, J=7 \mathrm{~Hz}, \mathrm{H}-26), 0.84(3 \mathrm{H}, \mathrm{m}, \mathrm{H}-29)$, $0.88(3 \mathrm{H}, \mathrm{t}, J=6.5 \mathrm{~Hz}, \mathrm{H}-18), 0.92(3 \mathrm{H}, \mathrm{d}, J=$ $7 \mathrm{~Hz}, \mathrm{H}-21), 1.03(3 \mathrm{H}, \mathrm{s}, \mathrm{H}-19), 1.25-1.38(14 \mathrm{H}$, $\mathrm{m}, \mathrm{H}-4^{\prime}, 5^{\prime}, 6^{\prime}, 7^{\prime}, 15^{\prime}, 16$ ', 17'), 1.59-1.65 (2H, m, H-3'), 2.03-2.08 (4H, m, H-8', 14'), 2.29 (2H, t, $J=$ $\left.7.5 \mathrm{~Hz}, \mathrm{H}-2^{\prime}\right), 2.76\left(2 \mathrm{H}, \mathrm{t}, J=6.5 \mathrm{~Hz}, \mathrm{H}-11^{\prime}\right), 4.6$ $(1 \mathrm{H}, \mathrm{m}, \mathrm{H}-3), 5.30-5.40(5 \mathrm{H}, \mathrm{m}$, olefinic protons $)$. $-{ }^{13} \mathrm{C} \mathrm{NMR}\left(\mathrm{CDCl}_{3}, 500 \mathrm{MHz}\right): \delta_{\mathrm{C}}=11.8(\mathrm{C}-18)$, 11.9 (C-29), 14.1 (C-18'), 18.8 (C-21), 19.0 (C-27), 19.3 (C-19), 19.8 (C-26), 21.0 (C-11), 22.7 (C-17'), 23.1 (C-28), 24.3 (C-15), 25.1 (C-3'), 25.6 (C-11'), 26.0 (C-23), 27.2 (C-8',14'), 27.8 (C-2), 28.3 (C16), 29.1 (C-25), 29.2 (C-4'), 29.3 (C-5'), 29.4 (C15'), 29.6 (C-6'), 29.7 (C-7'), 31.6 (C-16'), 31.8 
(C-7), 31.9 (C-8), 33.9 (C-22), 34.4 (C-2'), 36.2 (C-20), 36.5 (C-10), 37.0 (C-1), 38.2 (C-4), 39.7 (C-12), 42.3 (C-13), 45.8 (C-24), 50.0 (C-9), 56.0 (C-17), 56.7 (C-14), 73.7 (C-3), 122.6 (C-6), 127.9 (C-10'), 128.0 (C-12'), 130.0 (C-9'), 130.2 (C-13'), 139.7 (5), 173.3 (C=O). - EI-MS: $m / z(\%)=676$ $[\mathrm{M}]^{+}(1), 396$ (100), 381 (15), 288 (12), 275 (14), 255 (16), 213 (12).

$\beta$-Sitosterol (3): $R_{\mathrm{f}}=0.13$ in $n$-hexane/EtOAc $(9: 1 \mathrm{v} / \mathrm{v}) .-{ }^{1} \mathrm{H}$ NMR $\left(\mathrm{CDCl}_{3}, 500 \mathrm{MHz}\right): \delta_{\mathrm{H}}=0.68$ (3H, s, H-18), 0.79 (3H, d, $J=6.5 \mathrm{~Hz}, \mathrm{H}-27), 0.82$ $(3 \mathrm{H}, \mathrm{d}, J=6.5 \mathrm{~Hz}, \mathrm{H}-26), 0.83(3 \mathrm{H}, \mathrm{t}, J=7.5 \mathrm{~Hz}$, H-29), 0.91 (3H, d, $J=6.5 \mathrm{~Hz}, \mathrm{H}-21), 0.99$ (3H, s, $\mathrm{H}-19), 3.5$ (1H, m, H-3), 5.3 (1H, brd, $J=5 \mathrm{~Hz}$, $\mathrm{H}-6) .-{ }^{13} \mathrm{C} \mathrm{NMR}\left(\mathrm{CDCl}_{3}, 500 \mathrm{MHz}\right): \delta_{\mathrm{C}}=11.8$ (C-18), 11.9 (C-29), 18.8 (C-21), 19.0 (C-27), 19.4 (C-19), 19.8 (C-26), 21.1 (C-11), 23.0 (C-28), 24.3 (C-15), 26.0 (C-23), 28.3 (C-16), 29.1 (C-25), 31.6 (C-2), 31.8 (C-7), 31.9 (C-8), 33.9 (C-22), 36.1 (C20), 36.5 (C-10), 37.2 (C-1), 39.7 (C-12), 42.3 (C13), 45.8 (C-24), 50.1 (C-9), 56.0 (C-17), 56.8 (C14), 71.8 (C-3), 121.7 (C-6), 140.7 (C-5). - EI-MS: $m / z(\%)=414[\mathrm{M}]^{+}(100), 399(38), 396(32), 381$ (40), 303 (75), 273 (37), 231 (41), 213 (67).

Uvaol (12-ursen-3 $\beta, 28$-diol) (4): $R_{\mathrm{f}}=0.23$ in $n$-hexane/EtOAc $(8: 2 \mathrm{v} / \mathrm{v}) .-{ }^{1} \mathrm{H} \mathrm{NMR}\left(\mathrm{CDCl}_{3}\right.$, $500 \mathrm{MHz}): \delta_{\mathrm{H}}=0.80(3 \mathrm{H}, \mathrm{s}, \mathrm{H}-25), 0.81(3 \mathrm{H}, \mathrm{d}$, $J=5.8 \mathrm{~Hz}, \mathrm{H}-30), 0.94$ (3H, d, H-29), 0.95 (3H, s, H-24), 0.99 (3H, s, H-26), 1.00 (3H, s, H-27), 1.11 (3H, s, H-23), 3.19 (1H, d, $J=11 \mathrm{~Hz}, \mathrm{H}-28 \mathrm{a}), 3.23$ $(1 \mathrm{H}, \mathrm{dd}, J=4.9,11.3 \mathrm{~Hz}, \mathrm{H}-3), 3.53$ (1H, d, $J=$ $11 \mathrm{~Hz}, \mathrm{H}-28 \mathrm{~b}), 5.14$ (1H, t, $J=3.5, \mathrm{H}-12) .-{ }^{13} \mathrm{C}$ NMR $\left(\mathrm{CDCl}_{3}, 500 \mathrm{MHz}\right): \delta_{\mathrm{C}}=15.6(\mathrm{C}-24), 15.7$ (C-25), 16.8 (C-29), 17.3 (C-26), 18.3 (C-6), 21.3 (C-30), 23.3 (C-27), 23.4 (C-11), 26.0 (C-16), 27.2 (C-2), 28.1 (C-23), 29.7 (C-15), 30.6 (C-21), 32.8 (C-7), 35.2 (C-22), 36.8 (C-17), 38.0 (C-10), 38.8 (C-1), 38.8 (C-4), 39.3 (C-19), 39.4 (C-20), 40.0 (C8), 42.8 (C-14), 47.6 (C-9), 54.0 (C-18), 55.1 (C-5), 69.9 (C-28), 79.0 (C-3), 125.0 (C-12), 138.7 (C-13). - EI-MS: $m / z(\%)=442[\mathrm{M}]^{+}(5), 411$ (35), 234 (42), 203 (100), 189 (42), 133 (29).

Eugenol (5): $R_{\mathrm{f}}=0.26$ in $n$-hexane $/ \mathrm{CHCl}_{3}(3: 7$ v/v). $-{ }^{1} \mathrm{H} \mathrm{NMR}\left(\mathrm{CDCl}_{3}, 500 \mathrm{MHz}\right): \delta_{\mathrm{H}}=3.32(2 \mathrm{H}$, $\mathrm{d}, J=6.65 \mathrm{~Hz}, \mathrm{H}-7), 3.87\left(3 \mathrm{H}, \mathrm{s}, \mathrm{OCH}_{3}\right), 5.05(2 \mathrm{H}$, m, H-9), 5.48 (1H, brs, OH), 5.95 (1H, m, H-8), 6.69 (2H, m, H-3 and H-5), $6.84(1 \mathrm{H}, \mathrm{d}, J=8.5 \mathrm{~Hz}$, $\mathrm{H}-6) .-{ }^{13} \mathrm{C} \mathrm{NMR}\left(\mathrm{CDCl}_{3}, 500 \mathrm{MHz}\right): \delta_{\mathrm{C}}=39.9(\mathrm{C}-$ 7), $55.8\left(\mathrm{OCH}_{3}\right), 111.1$ (C-3), 114.2 (C-6), 115.5 (C-9), 121.2 (C-5), 131.9 (C-4), 137.8 (C-8), 143.9 (C-1), 146.4 (C-2).
Catechin (6): $R_{\mathrm{f}}=0.37$ in $\mathrm{CHCl}_{3} / \mathrm{MeOH}(8: 2$ $\mathrm{v} / \mathrm{v}) .-{ }^{1} \mathrm{H}$ NMR $\left(\mathrm{CD}_{3} \mathrm{OD}, 500 \mathrm{MHz}\right): \delta_{\mathrm{H}}=2.51$ (1H, dd, $J=16.1,8.2 \mathrm{~Hz}, \mathrm{H}-4 \mathrm{a}), 2.86$ (1H, dd, $J=$ 16.1, 5.4 Hz, H-4b), 3.97 (1H, m, H-3), 4.57 (1H, $\mathrm{d}, J=7.5 \mathrm{~Hz}, \mathrm{H}-2), 5.86(1 \mathrm{H}, \mathrm{d}, J=2.2 \mathrm{~Hz}, \mathrm{H}-8)$, $5.93(1 \mathrm{H}, \mathrm{d}, J=2.2 \mathrm{~Hz}, \mathrm{H}-6), 6.72(1 \mathrm{H}, \mathrm{dd}, J=8.1$, $2 \mathrm{~Hz}, \mathrm{H}-6$ ') 6.76 (1H, d, J = 8.1, H-5'), 6.84 (1H, d, $J=2 \mathrm{~Hz}, \mathrm{H}-2$ '). $-{ }^{13} \mathrm{C} \mathrm{NMR}\left(\mathrm{CD}_{3} \mathrm{OD}, 500 \mathrm{MHz}\right)$ : $\delta_{\mathrm{C}}=28.4(\mathrm{C}-4), 68.6(\mathrm{C}-3), 82.7(\mathrm{C}-2), 95.4(\mathrm{C}-8)$, 96.2 (C-6), 100.7 (C-10), 115.1 (C-2'), 116.0 (C-5'), 119.9 (C-6'), 132.0 (C-1'), 146.1 (C-3'), 146.1 (C4'), 156.8 (C-9), 157.4 (C-5), 157.6 (C-7).

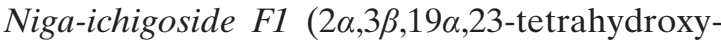
urs-12-en-28-oic acid $\beta$-D-glucopyranosyl ester) (7): $R_{\mathrm{f}}=0.47$ in $\mathrm{CHCl}_{3} / \mathrm{MeOH}(75: 25 \mathrm{v} / \mathrm{v}) .-{ }^{1} \mathrm{H}$ NMR ( $\left.\mathrm{CD}_{3} \mathrm{OD}, 500 \mathrm{MHz}\right): \delta_{\mathrm{H}}=0.7(3 \mathrm{H}, \mathrm{s}, \mathrm{H}-24)$, 0.78 (3H, s, H-26), 0.93 (3H, d, $J=6.5 \mathrm{~Hz}, \mathrm{H}-30)$, 1.04 (3H, s, H-25), 1.21 (3H, s, H-29), 1.34 (3H, s, H-27), 2.52 (1H, s, H-18), 3.25 (1H, d, $J=11 \mathrm{~Hz}$, H-23a), 3.49 (1H, d, $J=11 \mathrm{~Hz}, \mathrm{H}-23 \mathrm{~b}), 3.67$ (1H, $\mathrm{m}, \mathrm{H}-2), 3.35-3.82\left(5 \mathrm{H}, \mathrm{H}_{\mathrm{Glc}}-2-6\right), 5.31$ (2H, d, $\left.J=8.1 \mathrm{~Hz}, \mathrm{H}_{\mathrm{Glc}}-1, \mathrm{H}-12\right) .-{ }^{13} \mathrm{C} \mathrm{NMR}\left(\mathrm{CD}_{3} \mathrm{OD}\right.$, $500 \mathrm{MHz}): \delta_{\mathrm{C}}=13.9(\mathrm{C}-24), 16.6(\mathrm{C}-30), 17.6$ (C-26), 17.7 (C-25), 19.2 (C-6), 24.7 (C-27), 24.8 (C-11), 26.5 (C-16), 27.1 (C-29), 27.2 (C-21), 29.6 (C-15), 33.5 (C-7), 38.3 (C-22), 38.9 (C-10), 40.8 (C-8), 42.8 (C-14), 42.9 (C-20), 44.1 (C-4), 48.0 (C1), 48.2 (C-9), 48.4 (C-5), 54.9 (C-18), 66.4 (C-23), 69.7 (C-2), 73.6 (C-19), 78.3 (C-3), 129.7 (C-12), 139.5 (C-13), 178.5 (C=O); glucose: 62.4 (C-6'), 71.1 (C-4'), 73.8 (C-2') 78.3 (C-5'), 78.5 (C-3'), 95.8 (C-1'). - EI-MS: $m / z(\%)=504\left[\mathrm{M}-\mathrm{C}_{6} \mathrm{H}_{10} \mathrm{O}_{5}\right]$ (4), 426 (23), 344 (100), 264 (15), 246 (18), 239 (22), 219 (17), 201 (34), 164 (72).

Gein (eugenyl vicianoside) (8): $R_{\mathrm{f}}=0.2$ in $\mathrm{CHCl}_{3} / \mathrm{MeOH}(8: 2 \mathrm{v} / \mathrm{v}) .-{ }^{1} \mathrm{H} \mathrm{NMR},{ }^{13} \mathrm{C} \mathrm{NMR}$, and $\mathrm{HMBC}\left(\mathrm{CD}_{3} \mathrm{OD}, 500 \mathrm{MHz}\right)$ : see Table I.

Sucrose (9): $R_{\mathrm{f}}=0.28$ in EtOAc/MeOH (6:4 $\mathrm{v} / \mathrm{v}) .-{ }^{1} \mathrm{H}$ NMR $\left(\mathrm{D}_{2} \mathrm{O}, 500 \mathrm{MHz}\right): \delta_{\mathrm{H}}=3.31(1 \mathrm{H}$, $\left.\mathrm{t}, J=9.5 \mathrm{~Hz}, \mathrm{H}_{\mathrm{Glc}}-4\right), 3.4(1 \mathrm{H}, \mathrm{dd}, J=10,3.8 \mathrm{~Hz}$, $\left.\mathrm{H}_{\mathrm{Glc}}-2\right), 3.51\left(2 \mathrm{H}, \mathrm{s}, \mathrm{H}_{\mathrm{Fru}}-1\right), 3.59(1 \mathrm{H}, \mathrm{t}, J=9.5 \mathrm{~Hz}$, $\left.\mathrm{H}_{\mathrm{Glc}}-3\right), 3.66$ (4H, m, $\mathrm{H}_{\mathrm{Glc}}-6$ and $\left.\mathrm{H}_{\mathrm{Fru}}-6\right), 3.69(1 \mathrm{H}$, $\left.\mathrm{m}, \mathrm{H}_{\mathrm{Glc}}-5\right), 3.72\left(1 \mathrm{H}, \mathrm{m}, \mathrm{H}_{\mathrm{Fru}}-5\right), 3.89(1 \mathrm{H}, \mathrm{t}, J=$ $\left.8.5 \mathrm{~Hz}, \mathrm{H}_{\mathrm{Fru}}-4\right), 4.04\left(1 \mathrm{H}, \mathrm{d}, J=8.7 \mathrm{~Hz}, \mathrm{H}_{\mathrm{Fru}}-3\right)$, $5.25\left(1 \mathrm{H}, \mathrm{d}, J=3.8 \mathrm{~Hz}, \mathrm{H}_{\mathrm{Glc}}-1\right) .-{ }^{13} \mathrm{C} \mathrm{NMR}\left(\mathrm{D}_{2} \mathrm{O}\right.$, $500 \mathrm{MHz}): \delta_{\mathrm{C}}=60.1\left(\mathrm{C}_{\mathrm{Glc}}-6\right), 61.3\left(\mathrm{C}_{\mathrm{Fru}}-1\right), 62.3$ $\left(\mathrm{C}_{\mathrm{Fru}}-6\right), 69.2\left(\mathrm{C}_{\mathrm{Glc}}-4\right), 71.0\left(\mathrm{C}_{\mathrm{Glc}}-2\right), 72.4\left(\mathrm{C}_{\mathrm{Glc}}-5\right)$, $72.5\left(\mathrm{C}_{\mathrm{Glc}^{-}}-3\right), 73.9\left(\mathrm{C}_{\mathrm{Fru}}-4\right), 76.3\left(\mathrm{C}_{\mathrm{Fru}^{-}}-3\right), 81.3\left(\mathrm{C}_{\mathrm{Fru}^{-}}{ }^{-}\right.$ 5), $92.1\left(\mathrm{C}_{\mathrm{Glc}}-1\right), 103.6\left(\mathrm{C}_{\mathrm{Fru}}-2\right)$. 
Table I. NMR data of compound $\mathbf{8}$ in $\mathrm{CD}_{3} \mathrm{OD}$.

\begin{tabular}{|c|c|c|c|}
\hline Carbon No. & ${ }^{13} \mathrm{C}$ NMR (ppm) & ${ }^{1} \mathrm{H}$ NMR (ppm) & HMBC \\
\hline 1 & 146.1 & & H-3, H-5, H-6, H-1, \\
\hline 2 & 150.7 & & $\mathrm{OCH}_{3}, \mathrm{H}-3, \mathrm{H}-6$ \\
\hline 3 & 114.0 & $6.82(\mathrm{~d}, \mathrm{~J}=2 \mathrm{~Hz}, 1 \mathrm{H})$ & $\mathrm{H}-5, \mathrm{H}-7$ \\
\hline 4 & 136.4 & & H-3, H-5, H-6, H-7, H-8 \\
\hline 5 & 122.2 & $6.76(\mathrm{dd}, \mathrm{J}=8.2,2 \mathrm{~Hz}, 1 \mathrm{H})$ & $\mathrm{H}-3, \mathrm{H}-7$ \\
\hline 6 & 118.3 & $7.11(\mathrm{~d}, \mathrm{~J}=8.1 \mathrm{~Hz}, 1 \mathrm{H})$ & \\
\hline 7 & 40.7 & $3.33(\mathrm{~d}, \mathrm{~J}=6.6 \mathrm{~Hz}, 2 \mathrm{H})$ & H-3, H-5, H-8, H-9 \\
\hline 8 & 139.0 & $5.95(\mathrm{~m}, 1 \mathrm{H})$ & $\mathrm{H}-7$ \\
\hline 9 & 115.8 & $5.04(\mathrm{~m}, 2 \mathrm{H})$ & $\mathrm{H}-7$ \\
\hline $\mathrm{OMe}$ & 56.6 & $3.84(\mathrm{~s}, 3 \mathrm{H})$ & \\
\hline \multicolumn{4}{|l|}{ Glc } \\
\hline 1 ' & 102.7 & $4.86(\mathrm{~d}, \mathrm{~J}=7.1 \mathrm{~Hz}, 1 \mathrm{H})$ & \\
\hline 2' & 74.9 & $3.48(\mathrm{~m}, 1 \mathrm{H})$ & \\
\hline 3 , & 77.6 & $3.46(\mathrm{~m}, 1 \mathrm{H})$ & H-4, \\
\hline 4' & 71.4 & $3.40(\mathrm{~m}, 1 \mathrm{H})$ & \\
\hline 5 & 77.3 & $3.58(\mathrm{~m}, 1 \mathrm{H})$ & H-4, H-6' \\
\hline \multirow[t]{2}{*}{ 6' } & 69.1 & $3.75(\mathrm{~m}, 1 \mathrm{H})$ & H-4', H-1" \\
\hline & & $4.08(\mathrm{dd}, \mathrm{J}=11.5,2.1 \mathrm{~Hz}, 1 \mathrm{H})$ & \\
\hline \multicolumn{4}{|c|}{ (2) } \\
\hline $1 ”$ & 104.7 & $4.28(\mathrm{~d}, \mathrm{~J}=6.8 \mathrm{~Hz}, 1 \mathrm{H})$ & Н-6’a, H- 6’b, H-2", H-5"a, H-5"b \\
\hline $2 "$ & 72.4 & $3.56(\mathrm{~m}, 1 \mathrm{H})$ & \\
\hline $3 "$ & 74.1 & $3.48(\mathrm{~m}, 1 \mathrm{H})$ & H-1", H-2", H-4" \\
\hline $4 "$ & 69.4 & $3.76(\mathrm{~m}, 1 \mathrm{H})$ & H-5"a, H-5"b \\
\hline \multirow[t]{2}{*}{$5 "$} & 66.6 & $3.44(\mathrm{~m}, 1 \mathrm{H})$ & H-1" \\
\hline & & $3.81(\mathrm{dd}, \mathrm{J}=12.3,3.3 \mathrm{~Hz}, 1 \mathrm{H})$ & \\
\hline
\end{tabular}

\section{Results}

The inhibition zone diameters (IZD) of test samples and antibiotics against $H$. pylori are summarized in Tables II and III. No inhibition zone was observed for DMSO in control plates. The MIC values of the aqueous fraction and ciprofloxacin (as a positive control) against several Gram-positive and Gram-negative bacteria are shown in Table IV.

From the EtOAc and $\mathrm{MeOH}$ extracts of the roots, nine compounds including two triterpenoids, uvaol (4) (Mahato and Kundu, 1994) and niga-ichigoside F1 (7) (Bowen-Forbes et al., 2009), three sterols, $\beta$-sitosterol (3), $\beta$-sitosteryl acetate (1) (Goad and Akihisa, 1997), and $\beta$-sitosteryl linoleate (2) (Huh et al., 2010; Dyas et al., 1991), one phenyl propanoid, eugenol (5), one phenolic glycoside, gein (8) (Takeda et al., 1998; Shimoda et al., 2007; Shigenaga et al., 1985), one flavanol, (+)-catechin (6) (Banavides et al., 2006), and sucrose (9) (Agrawal, 1992) were isolated by column chromatography and identified by comparison of their spectroscopic data with those in the literature (Fig. 1). Spectroscopic data such as ${ }^{1} \mathrm{H}$ and ${ }^{13} \mathrm{C}$ NMR, HMQC, HMBC, ${ }^{1} \mathrm{H}-{ }^{1} \mathrm{H}$ COSY and EI-MS were employed for identification of the isolated compounds. In the previous study, the NMR data of gein were reported in $\mathrm{CDCl}_{3}$ and pyridine- $\mathrm{d}_{5}$ (Shigenaga et al., 1985). To the best of our knowledge, there is no report on the 2DNMR correlations of this compound, so that this is the first report on the NMR data of gein in $\mathrm{CD}_{3} \mathrm{OD}$ and HSQC and HMBC correlations (Table I and Fig. 2).

\section{Discussion}

The $\mathrm{MeOH}$ extract of $G$. iranicum was considered an effective extract against one of the clinical isolates of $H$. pylori which was resistant to metronidazole. As shown in Table II, the chloroform fraction, obtained from the $\mathrm{MeOH}$ extract, was not effective against $H$. pylori, but the aqueous fraction showed a potent activity (Table II). Subfractions of the aqueous part were evaluated for their antibacterial activity against all isolates. Fraction D was the only one that displayed antibacterial activity $(\mathrm{MIC}=25 \mu \mathrm{g} / \mathrm{ml})$. Therefore, the fractions obtained from $\mathrm{D}$ fractionation were 
Table II. Inhibition zone diameters $(\mathrm{mm})$ of some extracts of G. iranicum.

\begin{tabular}{|c|c|c|c|c|c|c|c|c|c|c|c|c|}
\hline \multirow[t]{3}{*}{ Sample } & \multicolumn{12}{|c|}{ Mean inhibition zone diameter $[\mathrm{mm}]^{\mathrm{a}}$} \\
\hline & \multicolumn{3}{|c|}{$100 \mu \mathrm{g} / \mathrm{ml}$} & \multicolumn{3}{|c|}{$50 \mu \mathrm{g} / \mathrm{ml}$} & \multicolumn{3}{|c|}{$25 \mu \mathrm{g} / \mathrm{ml}$} & \multicolumn{3}{|c|}{$12.5 \mu \mathrm{g} / \mathrm{ml}$} \\
\hline & Is. $1^{\mathrm{b}}$ & Is.2 & Is. 3 & Is.1 & Is. 2 & Is.3 & Is.1 & Is. 2 & Is.3 & Is.1 & Is. 2 & Is.3 \\
\hline $\begin{array}{l}\text { Ethyl ac- } \\
\text { etate extract }\end{array}$ & - & - & - & - & - & - & - & - & - & - & - & - \\
\hline $\begin{array}{l}\text { Methanol } \\
\text { extract }\end{array}$ & $15 \pm 0.5$ & - & - & - & - & - & - & - & - & - & - & - \\
\hline $\begin{array}{l}\text { Chloroform } \\
\text { fraction }\end{array}$ & - & - & - & - & - & - & - & - & - & - & - & - \\
\hline $\begin{array}{l}\text { Aqueous } \\
\text { fraction }\end{array}$ & $35 \pm 0.5$ & $30 \pm 0.5$ & $24 \pm 0.5$ & $28 \pm 0$ & $26 \pm 0.5$ & $18 \pm 0.25$ & $24 \pm 0.57$ & $24 \pm 0.5$ & $16 \pm 0$ & $20 \pm 0.5$ & $19 \pm 0.5$ & $14 \pm 0$ \\
\hline Fraction D & $22 \pm 0.5$ & $21 \pm 0.25$ & $12 \pm 0.5$ & $15 \pm 0.5$ & $12 \pm 0.5$ & $10 \pm 0.5$ & $12 \pm 0$ & $10 \pm 0.5$ & - & - & - & - \\
\hline Fraction $\mathrm{D}_{4}$ & $17 \pm 0.25$ & $16 \pm 0.5$ & $20 \pm 0$ & $12 \pm 0.57$ & $12 \pm 0.5$ & $10 \pm 0.5$ & $10 \pm 0.5$ & $10 \pm 0.28$ & - & $10 \pm 0.25$ & $9 \pm 0$ & - \\
\hline Eugenol & $20 \pm 0.5$ & $22 \pm 0.28$ & $10 \pm 0.5$ & - & - & - & - & - & - & - & - & - \\
\hline Catechin & - & - & - & - & - & - & - & - & - & - & - & - \\
\hline $\begin{array}{l}\text { Niga-ichigo- } \\
\text { side F1 }\end{array}$ & - & - & - & - & - & - & - & - & - & - & - & - \\
\hline Gein & - & - & - & - & - & - & - & - & - & - & - & - \\
\hline
\end{tabular}

a The results are shown as mean $\pm \mathrm{SD}(n=3)$.

b Is, clinical isolate of $H$. pylori.

Table III. Inhibition zone diameters ( $\mathrm{mm}$ ) of some antibiotics against clinical isolates of $H$. pylori.

\begin{tabular}{|c|c|c|c|}
\hline \multirow[t]{2}{*}{ Antibiotic $[\mu \mathrm{g} / \mathrm{ml}]$} & \multicolumn{3}{|c|}{ Inhibition zone diameter $[\mathrm{mm}]$} \\
\hline & Is. $1^{\mathrm{a}}$ & Is. 2 & Is.3 \\
\hline \multicolumn{4}{|c|}{ Furazolidone (MIC $0.5 \mu \mathrm{g} / \mathrm{ml}$ ) } \\
\hline 2 & 34 & 19 & 25 \\
\hline 1 & 22 & 15 & 21 \\
\hline 0.5 & 11 & 10 & 18 \\
\hline 0.25 & - & - & 13 \\
\hline \multicolumn{4}{|c|}{ Tetracycline (MIC $2 \mu \mathrm{g} / \mathrm{ml}$ ) } \\
\hline 4 & 20 & - & - \\
\hline 2 & 13 & - & - \\
\hline 1 & - & - & - \\
\hline 0.5 & - & - & - \\
\hline \multicolumn{4}{|c|}{ Amoxicillin (MIC $0.5 \mu \mathrm{g} / \mathrm{ml}$ ) } \\
\hline 2 & 40 & 30 & 36 \\
\hline 1 & 30 & 24 & 25 \\
\hline 0.5 & 20 & 12 & 15 \\
\hline 0.25 & 11 & - & 10 \\
\hline \multicolumn{4}{|c|}{ Clarithromycin (MIC $2 \mu \mathrm{g} / \mathrm{ml})$} \\
\hline 4 & - & 35 & 33 \\
\hline 2 & - & 28 & 27 \\
\hline 1 & - & 24 & 24 \\
\hline 0.5 & - & 21 & 19 \\
\hline \multicolumn{4}{|c|}{ Metronidazole (MIC $8 \mu \mathrm{g} / \mathrm{ml}$ ) } \\
\hline 32 & - & - & - \\
\hline 16 & - & - & - \\
\hline 8 & - & - & - \\
\hline 4 & - & - & - \\
\hline
\end{tabular}

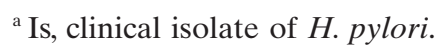

Table IV. Minimum inhibitory concentrations of the aqueous fraction of G. iranicum against several Grampositive and Gram-negative bacteria.

\begin{tabular}{|c|c|c|}
\hline \multirow[t]{2}{*}{ Bacterial strain $^{\mathrm{a}}$} & \multicolumn{2}{|c|}{ Minimum inhibitory concentration } \\
\hline & $\begin{array}{l}\text { Aqueous fraction } \\
{[\mathrm{mg} / \mathrm{ml}]}\end{array}$ & $\begin{array}{c}\text { Ciprofloxacin } \\
{[\mu \mathrm{g} / \mathrm{ml}]}\end{array}$ \\
\hline S. aureus & 16 & 0.19 \\
\hline S. epidermidis & 16 & 0.39 \\
\hline B. subtilis & 16 & 0.19 \\
\hline E. coli & 32 & 0.013 \\
\hline P. aeruginosa & 32 & 0.39 \\
\hline
\end{tabular}

evaluated again and resulted in one active fraction $\mathrm{D}_{4}$ (Table II). Finally, the isolated compounds from the chloroform and aqueous fractions (eugenol, gein, catechin, and niga-ichigoside F1) were examined for anti-H. pylori activity. Among the tested compounds, eugenol (5), isolated from the chloroform fraction, showed an antibacterial effect at a concentration of $100 \mu \mathrm{g} / \mathrm{ml}$ with inhibition zones of 20,22 , and $10 \mathrm{~mm}$ against the isolates 1,2, and 3 respectively. Eugenol is a major compound in the root oil of G. iranicum (Shahani et al., 2011) and is employed as a flavour- 
<smiles>CC[C@H](CC[C@@H](C)[C@H]1CCC2C3CC=C4C[C@@H](OC(=O)O)CC[C@]4(C)C3CC[C@]21C)C(C)C</smiles>

1: $\mathrm{R}=\mathrm{Ac}$

2: $\mathrm{R}=$ Linoleate 3: $\mathrm{R}=\mathrm{H}$<smiles>C=CCc1ccc(OC(=O)c2ccccc2)c(OC)c1</smiles>

5: $\mathrm{R}=\mathrm{H}$

8: $\mathrm{R}=\alpha$-L-Ara $(1 \rightarrow 6)-\beta-\mathrm{D}-\mathrm{Glc}$<smiles>Oc1cc(O)c2c(c1)O[C@H](c1ccc(O)c(O)c1)[C@H](O)C2</smiles>

6

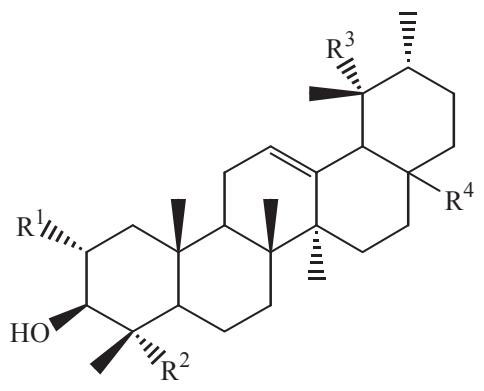

$$
\begin{array}{clll}
\mathrm{R}^{1} & \mathrm{R}^{2} & \mathrm{R}^{3} & \mathrm{R}^{4} \\
\text { 4: } \mathrm{H} & \mathrm{CH}_{3} & \mathrm{H} & \mathrm{CH}_{2} \mathrm{OH} \\
\text { 7: } \mathrm{OH} & \mathrm{CH}_{2} \mathrm{OH} & \mathrm{OH} & \text { COOGLc }
\end{array}
$$

Fig. 1. Chemical structures of the isolated compounds.

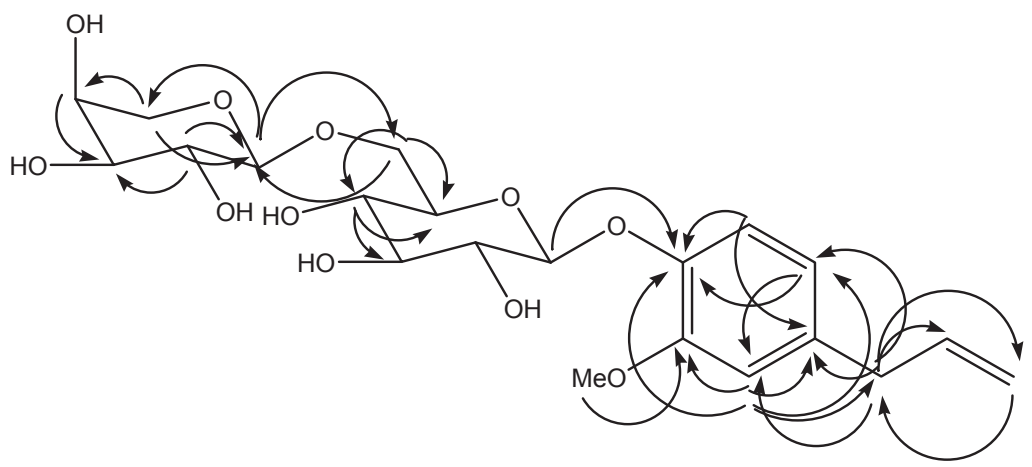

Fig. 2. HMBC correlations of compound $8(H \rightarrow C)$.

ing agent in cosmetics and food products, as well as a cement material in dentistry (Atsumi et al., 2005). Furthermore, eugenol has been found to exhibit a broad range of biological activities including antibacterial (Devi et al., 2010), antifungal (Campaniello et al., 2010), antiviral (Benencia and Courreges, 2000), antioxidant (Atsumi et al., 2005), anti-inflammatory (Yogalakshmi et al., 2010), anti nociceptive (Daniel et al., 2009), and antidepressant activity (Tao et al., 2005). A literature review shows that the minimal bactericidal concentration (MBC) of eugenol (obtained from essential oils) was $100 \mu \mathrm{g} / \mathrm{ml}$ against one strain of H. pylori, which had been isolated from a pa- 
tient with non-ulcer dyspepsia (Bergonzelli et al., 2003). Elsewhere it was reported that eugenol (commercial preparation) inhibited the growth of 30 strains of $H$. pylori at a concentration of $2 \mu \mathrm{g} /$ $\mathrm{ml}$ (Ali et al., 2005). Our antibacterial data for eugenol agree with the results reported by Bergonzelli et al. (2003).

Diglycosylated eugenol, named gein (8), produced no inhibition zone. Gein has been isolated from other Geum species (Shigenaga et al., 1985), and no biological activity has been reported for this compound. It seems that glycosylation of eugenol causes a decrease in anti- $H$. pylori activity. Also other purified compounds like catechin (6) and niga-ichigoside F1 (7) did not display any antibacterial activity at all concentrations.

Fraction $\mathrm{D}_{4}$ exhibited antibacterial activity as shown in Table II. Further investigation of fraction $\mathrm{D}_{4}$ indicated that this fraction can precipitate a solution of gelatin ( $1 \%)$, containing sodium chloride $(10 \%)$, and give a blue-black precipitate with ferric chloride. Therefore, this fraction should include polar compounds, especially hydrolysable tannins (Evans, 2009). There is a report in the literature on the antibacterial activity of hydrolysable tannins derived from medicinal plants against H. pylori (Funatogawa et al., 2004). For this reason, it seems that tannins from G. iranicum might

Abutorabi H. (2001), Ethnobotanical and phytochemical study on the plants of Rouin region. Pharm. D. Thesis. Faculty of Pharmacy, Tehran University of Medical Sciences, Tehran, Iran.

Agrawal P. K. (1992), NMR spectroscopy in the structural elucidation of oligosaccharides and glycosides. Phytochemistry 31, 3307-3330.

Ali S. M., Khan A. A., Ahmed I., Musaddiq M., Ahmed K. S., Polasa H., Venkateswar Rao L., Habibullah C. M., Sechi L. A., and Ahmed N. (2005), Antimicrobial activities of eugenol and cinnamaldehyde against the human gastric pathogen Helicobacter pylori. Ann. Clin. Microbiol. Antimicrob. 4, 1-7.

Atsumi T., Fujisawa S., and Tonosaki K. (2005), A comparative study of the antioxidant/prooxidant activities of eugenol and isoeugenol with various concentrations and oxidation conditions. Toxicol. In Vitro 19, 1025-1033.

Banavides A., Montoro P., Bassarello C., Piacente S., and Pizza C. (2006), Catechin derivatives in Jatropha macrantha stems: characterization and LC/ESI/MS/ MS quali-quantitative analysis. J. Pharm. Biomed. Anal. 40, 639-647. be one of the important groups of compounds active against $H$. pylori.

The aqueous fraction exhibited a moderate inhibitory activity against both Gram-positive and Gram-negative bacteria. The Gram-positive bacteria were inhibited at $16 \mathrm{mg} / \mathrm{ml}$, while Gram-negative bacteria were inhibited at about $32 \mathrm{mg} / \mathrm{ml}$.

In conclusion, considering the increasing resistance of $H$. pylori to antibiotics like metronidazole and clarithromycin, there is great interest in finding new drugs from natural sources. The results of this study show that $G$. iranicum has a significant antibacterial activity against resistant clinical isolates of $H$. pylori. Eugenol is one of the effective compounds, whereas other purified compounds (like gein, catechin, and niga-ichigoside F1) were not effective. Finally, the tannin-containing fraction of $G$. iranicum showed considerable antiH. pylori activity. Further investigation is required to purify the active tannins from this plant.

\section{Acknowledgement}

This research has been supported by a grant from Tehran University of Medical Sciences and Health Services. The authors thank Mr. Yousef Ajani from the Institute of Medicinal Plants for plant collection and identification.

Benencia F. and Courreges M. C. (2000), In vitro and in vivo activity of eugenol on human herpes virus. Phytother. Res. 14, 495-500.

Bergonzelli G. E., Donnicola D., Porta N., and CorthésyTheulaz I. E. (2003), Essential oils as components of a diet-based approach to management of Helicobacter infection. Antimicrob. Agents Chemother. 47, 3240-3246.

Bowen-Forbes C. S., Mulabagal V., Liu Y., and Nair M. G. (2009), Ursolic acid analogues: non-phenolic functional food components in Jamaican raspberry fruits. Food Chem. 116, 633-637.

Campaniello D., Corbo M. R., and Sinigaglia M. (2010), Activity of eugenol against Penicillium, Aspergillus and Fusarium species. J. Food Prot. 73, 1124-1128.

Daniel A. N., Sartoretto S. M., Schmidt G., CaparrozAssef S. M., Bersani-Amado C. A., and Cuman R. K. N. (2009), Anti-inflammatory and antinociceptive activities of eugenol essential oil in experimental animal models. Braz. J. Pharmacog. 19, 212-217.

Devi K. P., Nisha S. A., Sakthivel R., and Pandian S. K. (2010), Eugenol (an essential oil of clove) acts as an antibacterial agent against Salmonella typhi by dis- 
rupting the cellular membrane. J. Ethnopharmacol. 130, $107-115$.

Dyas L., Prescott M. C., Evershed R. P., and Goad L. J. (1991), Steryl esters in a cell suspension culture of celery (Apium graveolens). Lipids 26, 536.

Evans W. C. (2009), Trease and Evans Pharmacognosy, $16^{\text {th }}$ ed. Saunders Elsevier, Edinburgh.

Faramarzi M. A., Moghimi M., Monsef-Esfahani H. R., Shahverdi A. R., and Khodaee S. (2008), Chemical composition and antimicrobial activity of essential oils from Geum kokanicum. Chem. Nat. Compd. 44, $811-813$

Fauci A. S., Branuwald E., Kasper D. L., Hauser S. L., Longo D. L., Jameson J. L., and Loscalzo J. (2008), In: Harrison's Principles of Internal Medicine, $17^{\text {th }}$ ed. Mc Graw-Hill, New York.

Funatogawa K., Hayashi S., Shimomura H., Yoshida T., Hatano T., Ito H., and Hirai Y. (2004), Antibacterial activity of hydrolysable tannins derived from medicinal plants against Helicobacter pylori. Microbiol. Immunol. 48, 251-261.

Goad L. J. and Akihisa T. (1997), Analysis of Sterols. Blackie Academic \& Professional, London.

Gruenwald J. (2004), PDR for Herbal Medicine. Thomson PDR, Montvale.

Huh S., Kim Y. S., Jung E., Lim J., Jung K. S., Kim M. O., Lee J., and Park D. (2010), Melanogenesis inhibitory effect of fatty acid alkyl esters isolated from Oxalis triangularis. Biol. Pharm. Bull. 33, 1242-1245.

Khatamsaz K. (1992), Flora of Iran, No. 6: Rosaceae. Research Institute of Forest and Rangelands, Karaj.

Khorramizdeh M. R., Shahverdi A. R., Saadat F., and Monsef-Esfahani H. R. (2006), Inhibitory effect of Geum kokanicum roots on matrix metalloproteinases expression. Pharmaceut. Biol. 44, 266-270.

Kurokawa M., Nagasaka K., Hirabayashi T., Uyama S., Sato H., Kageyama T., Kadota S., Ohyama H., Hozumi T., and Namba T. (1995), Efficacy of traditional herbal medicines in combination with acyclovir against herpes simplex virus type 1 infection in vitro and in vivo. Antiviral Res. 27, 19-37.

Mahato S. B. and Kundu A. P. (1994), ${ }^{13}$ C-NMR spectra of pentacyclic triterpenoids - complication and salient features. Phytochemistry 37, 1517-1573.

Mégraud F. (2004), H. pylori antibiotic resistance: prevalence, importance, and advances in testing. Gut 53, $1374-1384$.

Ming D. S., Xu H. X., Dong H., and But P. P. H. (2000), Research progress in chemical constituents and biological activities of Geum species. Acta Pharm. Sin. 35, 552-558.

Mozaffarian V. (1996), A Dictionary of Iranian Plant Names. Farhang Moaser Publication, Tehran.

Nariman F., Eftekhar F., Habibi Z., and Falsafi T. (2004), Anti-Helicobacter pylori activities of six Iranian plants. Helicobacter 9, 146-151.

NCCLS (2006), Methods for Dilution Antimicrobial Susceptibility Tests for Bacteria That Grow Aerobically. Approved Standard M7-A7, $7^{\text {th }}$ ed. National Committee for Clinical Laboratory Standards, Wayne, PA, USA.
Panizzi L., Catalano S., Miarelli C., Cioni P. L., and Campeol E. (2000), In vitro antimicrobial activity of extracts and isolated constituents of Geum rivale. Phytother. Res. 14, 561-563.

Russo A., Cardile V., Lombardo L., Vanella A., and Garbarino J. A. (2005), Antioxidant activity and antiproliferative action of methanolic extract of Geum quellyon sweet roots in human tumor cell lines. J. Ethnopharmacol. 100, 323-332.

Shahani S., Monsef-Esfahani H. R., Hajiaghaee R., and Gohari A. R. (2011), Chemical composition of essential oil and hydrolate of Geum iranicum Khatamsaz. J. Essent. Oil Res. 23, 29-33.

Shigenaga S., Kouno I., and Kawano N. (1985), Triterpenoids and glycosides from Geum japonicum. Phytochemistry $\mathbf{2 4}, 115-118$.

Shimoda K., Kwon S., Utsuki A., Ohiwa S., Katsuragi H., Yonemoto N., Hamada H., and Hamada H. (2007), Glycosylation of capsaicin and 8-nordihydrocapsaicin by cultured cells of Catharanthus roseus. Phytochemistry 68, 1391-1396.

Siavoshi F., Saniee P., Latifi-Navid S., Massarrat S., and Sheykholeslami A. (2010), Increase in resistance rates of $H$. pylori isolates to metronidazole and tetracycline - comparison of three 3-year studies. Arch. Iran Med. 13, 177-187.

Takeda Y., Ooiso Y., Masuda T., Honda G., Otsuka H., Sezik E., and Yesilada E. (1998), Iridoid and eugenol glycosides from Nepeta cadmea. Phytochemistry 49, 787-791.

Tao G., Irie Y., Li D. J., and Keung W. M. (2005), Eugenol and its structural analogs inhibit monoamine oxidase A and exhibit antidepressant-like activity. Bioorg. Med. Chem. 13, 4777-4788.

Tunón H., Olavsdotter C., and Bohlin L. (1995), Evaluation of anti-inflammatory activity of some Swedish medicinal plants. Inhibition of prostaglandin biosynthesis and PAF-induced exocytosis. J. Ethnopharmacol. 48, 61-76.

Vollmann C. and Schultze W. (1995), Composition of the root essential oils of several Geum species and related members of the subtribus Geinae (Rosaceae). Flavour Fragr. 10, 173-178.

Xu H. X., Ming D. S., Dong H., and But P. P. H. (2000), A new anti-HIV triterpene from Geum japonicum. Chem. Pharm. Bull. 48, 1367-1369.

Yogalakshmi B., Viswanathan P., and Anuradha C. V. (2010), Investigation of antioxidant, anti-inflammatory and DNA-protective properties of eugenol in thioacetamide-induced liver injury in rats. Toxicology 268, 204-212.

Yukawa T. A., Kurokawa M., Sato H., Yoshida Y., Kageyoma S., Hasegawa T., Namba T., Imakita M., Hozumi T., and Shiraki K. (1996), Prophylactic treatment of cytomegalovirus infection with traditional herbs. Antiviral Res. 32, 63-70.

Zeng F. Q., Xu H. X., Sim K. Y., Gunsekera R. M., and Cheu S. X. (1998), The anticoagulant effects of Geum japonicum extract and its constituents. Phytother. Res. 12, 146-148. 